\title{
Korpuskeln und das Ganze
}

Liebe Leserinnen, liebe Leser,

am Anfang der 60er-Jahre waren Juristen zumeist davon überzeugt, dass ihnen der Computer fremd ist. Gewiss, man hatte an der Universität Verwaltungsprobleme und dachte auch vorsichtig an eine umfassende Biographie der Rechtsliteratur - vorsichtig. Mehr Phantasie hingegen brachten die Computerhersteller auf. Ich erinnere mich, dass ich einmal mit einem Juraprofessor sprach und meinte, man könne doch das Rechtssystem dem Computer einprogrammieren. Bei auftretenden Rechtsfragen könnte man ihm die Fakten eingeben und ihn ein Urteil ermitteln lassen; das zumindest als Entscheidungshilfe. Der Professor war belustigt. Er nahm sich die Zeit und erklärte mir, warum dies vollkommen abwegig wäre; schon Aristoteles, sagte er, hätte bei einem solchen Ansinnen abgewinkt. Ich sah das ein. Seitdem ist mir, einem Ingenieur, der Unterschied zwischen Naturrecht und positivem Recht bekannt. Ich weiß: Der Richter darf, vor allem im Privatrecht, das Ganze nicht aus den Augen verlieren, muss in einer Verhandlung den ganzen Rechtsfall zu erfassen suchen und in allen seinem Zusammenhängen berücksichtigen.

Die antiken griechischen Naturphilosophen und danach vor allem Aristoteles, sahen die Welt und ihre Genese als ein Ganzes an, das aus Elementarem entstanden ist, nach Thales aus dem Wasser, nach Anaximenes aus der Luft etc. Doch gab es schon damals auch Denker wie Leukippos und Demokrit, die meinten, die Welt setze sich aus Atomen zusammen und werde von diesen kleinsten Teilchen her bestimmt. Solche unterschiedliche antike Sichtweisen übermittelten sich unserem Mittelalter. Zunächst in Form der ganzheitlich orientierten aristotelischen Philosophie. Seit Anbruch der Neuzeit finden auch die Atomisten wieder bei uns Zuspruch. Man zergliedert das Weltganze und entdeckt an den Teilen und ihren Zusammenhängen Naturgesetze. Dabei hält man sich positivistisch an Fakten und Beweise. Man will ferner auch heute nicht den Blick für das Ganze und seinen Ursprung verlieren und denkt diesbezüglich etwa über den Urknall nach. Wo die beiden gegensätzlichen Betrachtungsrichtungen aufeinandertreffen, offenbaren sie erkenntnistheoretische Probleme. Man fand z.B. an der elektromagnetischen Strahlung heraus, dass sie neben ihrem Wellen-Charakter auch Korpuskel-Charakter hat, dass also ihr eigentlicher Charakter uns letztlich verborgen ist.

Insbesondere das private Recht hat seinen Ursprung im angeborenen Gerechtigkeitsgefühl des Menschen, dem Naturrecht und seinen Rechtsprinzipien. Aber davon gibt es so viele Versionen wie es Personen und Rechtsfälle gibt; kein verbindliches Maßsystem. Das aber muss für den Gebrauch als positives Recht öffentlich festgelegt werden. Denn Rechtsgefühl und Naturrecht sind nicht festlegbar. Trotzdem schließen sie und das positives Recht sich nicht aus; vielmehr bedingen sie einander: Ohne Rechtsgefühl kein positives Recht und ohne positives Recht keine Bedienung des Rechtsgefühls. Es zeigt sich, dass das Ideal des Naturrechts und die Realität des positiven Rechts zwar - wie Welle und Korpuskel - unverträglich aber dennoch zweifaltig Eines sind.

Gleichwohl drängen Rechtsgefühl und Naturrecht auf eine fortschreitende Atomisierung des positiven Rechts. Es wächst, wird unübersichtlich und unhandlich. Die Atomisierung stößt an praktische Grenzen. Um dem Naturrecht darüber hinaus Geltung zu verschaffen, hat man zu seiner Absicherung besondere Freiheitsrechte formuliert, die Menschenrechte. Der Datenschutz spielt hier eine besondere Rolle, denn er schützt ein Menschenrecht, das Grundrecht auf informationelle Selbstbestimmung. In dieser Funktion kann er mit dem gerade geltenden positiven Recht - das ist sein Schicksal auf die Dauer nicht zufriedenstellend bedient werden. Das Rechtsgefühl - so wie es sich mit der Zeit und mit den Generationen ändert - will im positiven Recht immer wieder neu berücksichtigt werden. Das ist auch heute noch zu viel für den Computer.

Mit freundlichen Grüßen, Ihr

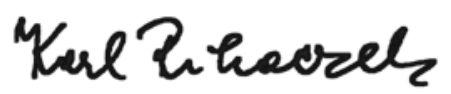

